\title{
ESTRATÉGIAS E INTERVENÇÕES PEDAGÓGICAS PARA APRENDIZAGEM DE MATEMÁTICA EM EDUCAÇÃO A DISTÂNCIA
}

\author{
Isolda Giani de Lima*
}

Laurete Zanol Sauer**

Resumo: Neste artigo apresentamos o relato e resultados de uma pesquisa realizada no Departamento de Matemática e Estatística da Universidade de Caxias do Sul, envolvendo estudantes de Cálculo Diferencial e Integral. A mesma constituiu-se, basicamente, na criação, utilização e análise de um ambiente de aprendizagem implementado e utilizado durante três edições do Programa em Educação à Distância para a Melhoria das Condições de Aprendizagem de Matemática. Realizamos uma investigação aplicada sobre a real possibilidade de fundamentar uma proposta pedagógica diferenciada, a partir da criação de estratégias que permitissem interferir na melhoria dos processos de ensino-aprendizagem de Matemática.

Buscamos desenvolver um modo educativo, através do qual fosse possível proporcionar condições de aprendizagem em ambiente que se diferenciasse dos que se caracterizam pela transmissão de informações e conteúdos, e primasse por ações reflexivas, por formas de pensar que propiciam a compreensão de conceitos e idéias matemáticas, através da qualidade das atividades promovidas e dos recursos utilizados.

Confirmamos essa possibilidade criando um ambiente para educação à distância incrementado por recursos que permitem a comunicação e a realização de atividades que promovem a construção de uma cultura informatizada e um saber cooperativo, por ações que privilegiam a interação.

Palavras-Chave: Aprendizagem de Matemática. Educação à distância. Ambientes de Aprendizagem. Interação.

Abstract: In this article we show the report and the results of a research performed at the Statistics and Mathematics Department of the University of Caxias do Sul, comprising students of Differential and Integral Calculus. This research is basically constituted of the creation, the usage and the analysis of a learning environment implemented during three editions of the Program on Education at Distance for the Improvement in the Conditions of Mathematics Learning. We performed an applied investigation about the real possibility of founding a differentiated pedagogical proposal, from the creation of strategies that permit to interfere in the improvement of the teaching-learning processes in Mathematics.

We searched for an educational way, through which it would be possible to promote learning conditions in an environment that is outstanding from those characterized by transmiting information and contents, and focused on reflexive actions and ways of thinking that promote the comprehension of Mathematical ideas and concepts through the quality of the activities promoted and the resources used.

We confirmed this possibility by the creation of a distance education learning environment incremented by resources that permit the communication and the performance of activities promoting the construction of a computer-aided culture and a cooperative knowledge where interaction is privileged.

\footnotetext{
* Professora do Departamento de Matemática e Estatística da Universidade de Caxias do Sul; Doutora em Informática na Educação; iglima@ucs.br.

** Professora do Departamento de Matemática e Estatística da Universidade de Caxias do Sul; Doutora em Informática na Educação; Izsauer@ucs.br.
} 
Keywords: Mathematics Learning, Education at Distance, Learning Environments, Interaction.

\section{Introdução}

A consciência sobre dificuldades com as quais professores e alunos se deparam ao lidarem com a Matemática, aliada à da necessidade de enfrentar essa questão, dada sua importância no contexto educacional, tem reflexos nos mais diversos setores da atividade humana e foi o desafio inicial, que motivou a implementação do projeto Mecam - Programa em Educação a Distância para a Melhoria das Condições de Aprendizagem de Matemática (SAUER, 2004). A possibilidade de utilização de ambientes de aprendizagem de Matemática, enriquecidos com recursos computacionais, tem sido discutida e questionada na comunidade acadêmica em geral e, com mais ênfase na de pesquisa educacional, tanto pelas dificuldades que ainda existem em relação à utilização da linguagem matemática em editores de texto comumente utilizados, quanto pela carência de experiências que confirmem a viabilidade de utilização de recursos telemáticos para educação a distância nessa área. Diante dessas considerações, compartilhamos neste artigo, a proposta oferecida pelo programa Mecam, através do qual foram planejadas e realizadas três edições do curso "Estudos Complementares de Cálculo Diferencial de Integral I", na modalidade à distância.

Fazem parte deste trabalho a análise e a discussão sobre condições de aprendizagem de Matemática e possibilidades de utilização de recursos telemáticos, levadas em consideração no planejamento e execução do programa, que passamos a descrever.

\section{Aprendizagem de Matemática em ambientes enriquecidos com recursos telemáticos}

A aprendizagem, não somente de Cálculo Diferencial e Integral, mas de Matemática, de modo geral, tem sido, ao longo dos anos, um problema para alunos e professores do grande número de cursos de graduação, em que a mesma é exigida. Ao mesmo tempo, a comunidade acadêmica anseia por proporcionar a seus cursos a qualidade necessária para que os egressos encontrem colocação no mercado de trabalho que exige, cada vez mais, habilidades de comunicação falada e escrita sobre idéias matemáticas que, em graus variados, estão envolvidas nas mais diversas áreas do conhecimento. Neste cenário, a utilização de recursos tecnológicos passou a ser considerada como uma alternativa metodológica com possibilidades para a superação de dificuldades relacionadas à aprendizagem, bem como para atender às expectativas relacionadas ao perfil e às habilidades dos egressos dos cursos da área de Ciências Exatas. Ao mesmo tempo, pesquisas (SKOVSMOSE, 2000; PONTE, 2003; VALENTINI, 2003; SAUER, 2004; LIMA, 2004; SOARES, 1997) sugerem que a qualidade do aprendizado depende, em grande parte, da qualidade das tarefas propostas aos alunos, das estratégias e intervenções pedagógicas e não, simplesmente, da disponibilidade ou do emprego de tecnologias computacionais.

Neste contexto de problematização, nossa preocupação foi, desde o início da pesquisa, com a formação de uma rede de interação, diálogo e cooperação para a construção de aprendizagens, o que vai muito além da escolha de um software ou da utilização do computador para transmitir informações e conteúdos. Compreendemos que algumas concepções de aprendizagem, ainda bastante presentes em ambientes educacionais, não suportam um fazer pedagógico que considere o estudante como tendo 
um caminho já percorrido, com conhecimentos já construídos, a partir dos quais, novas relações e novos objetos de conhecimento possam emergir da interação com o ambiente, sustentando a aprendizagem.

Conforme Piaget (1976), o conhecimento é construído na interação do sujeito com o objeto; é interagindo, a partir do que já é de seu domínio cognitivo e do que pretende incorporar, que o sujeito produz sua capacidade de conhecer, ao mesmo tempo em que produz o próprio conhecimento.

Não temos em educação soluções ideais, nem práticas pedagógicas definitivas, o que temos é um constante repensar sobre a própria ação que, como refere Maturana (1999), resulta do ato do viver do ser humano na construção da sua história. E, partindo do pressuposto da interação, buscamos fundamentar proposições educativas capazes de produzir aprendizagens, como efeitos de reflexões sobre ações realizadas, buscando sentido e compreensão para o fazer (LIMA, 2004). Considerando o ambiente de aprendizagem proposto, procuramos verificar como a interação em ambiente que articula ferramentas tecnológicas com uma metodologia específica, possibilita o desenvolvimento da aprendizagem de noções e conceitos matemáticos.

Na próxima sessão apresentamos as principais características do ambiente de aprendizagem para a realização do programa, cuja concepção está fundamentada na Epistemologia Genética de Jean Piaget, a partir de reflexões sobre condições de aprendizagem sob as quais esta se efetiva. São descritas as ações metodológicas, que privilegiam o diálogo, cujos elementos constitutivos comportam a ação e a reflexão e, consequentemente, a tomada de consciência e a aprendizagem, levadas a efeito no decorrer do programa.

\section{O ambiente de aprendizagem do Mecam}

O ambiente virtual de aprendizagem do Mecam foi construído com recursos tecnológicos idealizados, depurados e organizados de modo a permitir fácil acesso e flexibilidade de utilização, mesmo para aqueles alunos menos familiarizados com recursos da internet, de modo a não provocar embaraços tecnológicos que interferissem na realização dos estudos. Assim sendo, tais recursos constituiram-se apenas no que é necessário e indispensável para a realização do curso nesse programa em educação a distância.

A vida do ambiente é definida pelos fluxos de interações e de diálogos que surgem como frutos de estratégias e intervenções possibilitadas por um conjunto de ferramentas de apoio à comunicação, à colaboração e à realização de tarefas, disponibilizadas em páginas, que denominamos espaços, planejados e criados para atender aos objetivos do programa. Acrescentamos ao ambiente a utilização de um software matemático - Scientific Notebook, cuja opção justificou-se por sua qualidade como editor de textos que proporciona o manuseio da sintaxe matemática geral, tal como é usualmente expressa. Além disto, uma coleção de bibliotecas do software Maple permite agregar à edição de textos, processamentos matemáticos, sem a necessidade de conhecimento em programação. Esta qualidade é relevante, pois possibilita a participação de qualquer aluno, independentemente da sua desenvoltura em questões de informática.

Os diferentes espaços do ambiente são acessíveis por links, que identificam suas finalidades específicas. A organização e disponibilização dos mesmos são baseadas em princípios didáticos e psicopedagógicos que expressam nossa concepção da aprendizagem como algo que requer a participação ativa daquele que quer aprender. Entendemos que a participação ativa está diretamente relacionada a atividades de interação, colaboração e contribuição. Desta forma, o ambiente de aprendizagem foi 
organizado com o propósito de acompanhar o estudante e promover o desenvolvimento da autonomia e a capacidade de lidar com problemas e com tecnologia e de tomar decisões com autoconfiança.

Os espaços que constituem o ambiente do Mecam são os seguintes:

$\checkmark$ Dinâmica do Curso onde, além da apresentação do programa, é explicitada a sua metodologia, de forma a tornar claros para os alunos, os pressupostos teóricos e concepções de aprendizagem que a fundamentam, as orientações para a realização das atividades e para a participação efetiva no curso, além dos critérios de avaliação.

$\checkmark$ Agenda, espaço reservado para comunicações e avisos referentes ao desenvolvimento do curso e de interesse de todos os participantes.

$\checkmark$ Material de Apoio, onde são disponibilizadas orientações sobre a utilização de recursos tecnológicos, bem como respostas a Perguntas Freqüentes sobre questões tecnológicas. Para ambientes de aprendizagem de Matemática salientamos as características do software Scientific Notebook, que possibilitam a comunicação matemática em todas as suas formas de expressão, quais sejam, analítica, verbal, gráfica e numérica.

$\checkmark$ Leituras, destinado às referências bibliográficas sugeridas, bem como links de acesso a endereços relacionados aos temas de interesse do curso, além de Notas de Aula referentes aos tópicos da disciplina.

$\checkmark$ Discussões, que conta com um fórum que permite anexar arquivos, além de um recurso de Correio Interno para a troca de mensagens envolvendo outros assuntos, não especificamente relacionados às atividades do curso. A ferramenta de Correio Interno foi agregada ao ambiente, a partir da segunda edição, devido ao grande número de questionamentos no fórum, como trânsito simples de informações. Analisando a qualidade das discussões, concluímos que o objetivo específico de gerar conhecimento na forma de produção coletiva foi parcialmente atingido durante a primeira edição. Atribuímos isto ao fato de o fórum ter sido o único canal de discussão no ambiente. A grande quantidade de mensagens de caráter informativo ocasionou certa "poluição" e conseqüentemente dificuldades na utilização da ferramenta. Assim sendo, visando privilegiar no fórum discussões sobre questões matemáticas, a partir da segunda edição do programa, a ferramenta de correio interno foi integrada ao ambiente, o que possibilitou uma produção matemática de melhor qualidade do que a da primeira edição. É importante destacar que as produções geradas no fórum constituíram material de apoio aos estudos como Produções Coletivas na forma de tópicos ou hipertextos que organizam as produções dos próprios alunos, utilizando sua própria linguagem e que serviram de apoio às demais edições do mesmo curso, como um acervo que pode ser atualizado e implementado continuamente.

$\checkmark$ Alunos Participantes, constituído por um conjunto de pastas individuais, identificadas pelos nomes dos estudantes participantes e acessíveis pelas professoras responsáveis e pelos próprios alunos, mediante utilização de senhas. As referidas pastas - portfólio ou caderno do estudante - destinaram-se ao depósito de todos os materiais produzidos durante a realização dos estudos, incluindo os materiais de auto-avaliações e as fichas individuais de registro de desempenhos dos estudantes.

$\checkmark$ Avaliação, destinado ao acompanhamento dos estudantes em relação à realização das atividades propostas. Para tal, além de uma orientação detalhada sobre o processo de avaliação, um link - realização das atividades - apresenta, numa tabela resumo, a situação de cada atividade, e para cada estudante, como concluída, realizada parcialmente ou não realizada.

Três cursos foram realizados nesse ambiente, nos períodos acadêmicos: férias de verão, primeiro período regular e férias de inverno, sendo os ambientes próprios de cada edição disponibilizados, respectivamente, em: 
http://www.ucs.br/deme/disciplinas/mecam

http://www.ucs.br/deme/disciplinas/mecam2ed

http://www.ucs.br/deme/disciplinas/mecam3ed.

A utilização do ambiente em seus espaços específicos propiciou auxiliar os estudantes a minimizar dificuldades de aprendizagem, por uma prática de solicitar justificativas verbais para as resoluções apresentadas e uma análise da coerência das soluções obtidas, considerando o contexto no qual a questão estava inserida. Os estudos de aprimoramento dessa prática nos levaram ao método clínico de Piaget (Castorina et al.,1988), onde encontramos elementos de fundamentação para a proposta pedagógica, que consiste na integração de recursos tecnológicos e de uma metodologia de intervenção inspirada no referido método.

\section{A prática metodológica do Mecam}

A proposta metodológica norteadora do programa considera a investigação sobre as condições iniciais do aluno que ingressa no curso e a possibilidade de aprimorá-la, por um processo de reflexão e tomada de consciência que o leve à construção de conhecimentos e ao desenvolvimento de nova postura de autonomia e de responsabilidade por sua aprendizagem.

Com esse propósito, um conjunto de atividades, visando a (re)construção dos conceitos estruturais da disciplina é proposto para o desenvolvimento dos estudos e disponibilizado no ambiente, desde o início do curso. Cada atividade consta de um problema e um questionamento inicial que gera um processo de problematizações com a finalidade de identificar dificuldades e conhecimentos já construídos. Durante todo o trabalho, desde o princípio, o estudante é orientado a buscar auxílio na bibliografia recomendada, para apresentar, em cada momento, o que julgar ser o melhor, de acordo com sua condição.

Fazem parte do processo a análise e a discussão dos procedimentos adotados na resolução dos problemas e a proposição de novos questionamentos que utilizam erros ou acertos como fontes para o que precisa ser (re)elaborado a fim de que sejam superadas as dificuldades, ou como geradores de desafios que visem estabelecer novas relações e níveis mais elevados de compreensão.

Nos questionamentos e solicitação de justificativas, são sugeridas diferentes abordagens para as questões, com o propósito de auxiliar no entendimento dos significados. Em geral, os alunos iniciam com procedimentos algébricos - resolvendo uma equação ou aplicando uma fórmula, mas ao utilizarem outras formas como a simulação numérica, tradução da simbologia com as próprias palavras ou visualização geométrica, aumentam as possibilidades de compreensão.

Assim sendo, para cada aluno e em cada atividade, as propostas de estudo e o material de apoio têm caráter flexível, adequado a cada situação. As discussões, por sua vez, ocorrem em diferentes níveis, dependendo da necessidade, interesse e disponibilidade de cada um. Quanto ao tempo destinado às atividades, não consideramos prazos finais para o término de cada uma e sim uma data limite para a primeira apresentação de cada atividade. A partir desta, a elaboração das demais e os aperfeiçoamentos sugeridos para cada uma ocorrem simultaneamente. O prazo final para os aprimoramentos é agendado para uma semana antes do término do curso, quando os alunos se apresentam para a entrevista presencial. Nesta ocasião ocorre, também na presença do aluno e com sua concordância e devida justificativa, a confirmação da aprovação ou reprovação e o registro de sua avaliação em relação à proposta de aprendizagem que o curso oferece.

Uma primeira reunião presencial marca o início de cada curso. Nestes 
encontros procuramos fazer uma apresentação detalhada do programa, deixando claros os principais objetivos. A proposta metodológica adotada para atender tais objetivos é apresentada, salientando-se que a construção do conhecimento é de caráter individual e que o processo de aprendizagem exige querer aprender. De acordo com tais pressupostos, justificamos o desenvolvimento do curso a partir da realização de atividades que exigem a participação ativa do aluno em todos os momentos e a disposição para aprimorá-las, visando a compreensão dos conceitos, por exploração dos significados a partir da análise e discussão dos procedimentos adotados na resolução dos problemas.

Uma breve introdução ao software matemático Scientific Notebook também é tema do primeiro encontro, quando são esclarecidas dúvidas relacionadas ao download dos arquivos contendo as atividades, à utilização das pastas individuais e à participação no fórum, dentre outras. Visando a amenizar dificuldades com a tecnologia, também encaminhamos parte da primeira atividade priorizando noções básicas sobre a utilização ao software, como forma de orientar para a continuidade dos estudos $\mathrm{e}$ disponibilizamos, durante a primeira semana do curso, atendimentos presenciais para dúvidas de caráter tecnológico, além da monitoria virtual.

Ainda, no primeiro encontro, definimos em conjunto com os alunos, o calendário de apresentação da primeira e da última versão de cada uma das atividades programadas, estabelecendo a obrigatoriedade de dois aperfeiçoamentos para cada uma, demonstrados através das respostas dadas aos questionamentos, sugeridos visando resoluções adequadas e a compreensão do que está sendo discutido em cada caso. Entendemos que, desta forma, cada aluno pode, desde o início do curso, programar-se para o cumprimento de todas as tarefas solicitadas.

A aprovação no curso está vinculada à conclusão de todas as atividades com grau mínimo 1 , dentre os possíveis graus previstos $0,1,2,3$ e 4 , que definem os conceitos de desempenhos da Universidade, e à realização da entrevista presencial, onde o estudante apresenta argumentações sobre as respostas e justificativas apresentadas nos aperfeiçoamentos, confirmando ou elevando, caso aprimore mais uma vez as resoluções, o grau final de cada atividade. O grau final é 0 (zero) no caso de reprovação e é a média dos graus obtidos nos itens de avaliação global, quando aprovado.

Um ponto de destaque da metodologia está relacionado à possibilidade de identificar as dificuldades de cada aluno, sem necessariamente exigir a repetição de etapas já vencidas, mas, em cada caso, buscar soluções para os problemas detectados. Além disto, em qualquer processo de aprendizagem, aprende-se do que já se aprendeu, por reestruturação, reciclagem, até porque somos seres com passado, memória e sentido. (Demo, 2001).

Outro fator que destacamos como colaborador de aprendizagem no ambiente do Mecam, consiste na realização das atividades de auto-avaliação, promovidas visando a reflexão do estudante sobre o seu grau de envolvimento e desempenho no curso, além de coletar informações sobre dificuldades geradas pela "distância", sobre a metodologia, sobre possibilidades de estudar e aprender nesse ambiente. Algumas perguntas visaram promover a tomada de consciência sobre o processo individual de desenvolvimento, analisando o respectivo desempenho e procurando identificar e justificar, explicitando o assunto relacionado, as dificuldades encontradas e apontando possíveis ações que pudessem auxiliar a superá-las. Além disto, procuramos também, incentivá-los a apresentar todas as sugestões ou comentários julgados relevantes e que pudessem colaborar para melhorar as condições de sua aprendizagem.

As auto-avaliações foram propostas com a finalidade de promover a tomada de consciência das ações realizadas, ao levantarem questões relacionadas à assiduidade, interesse, tempo dedicado ao estudo, cumprimento de tarefas propostas, participação

$6 \longrightarrow$ V. 3 N $^{\circ}$ 2, Novembro, 2005 
com perguntas, respostas ou comentários visando melhorar a qualidade das aprendizagens, utilização da bibliografia sugerida, dos recursos disponibilizados pela Universidade, tais como biblioteca, laboratórios, monitoria. Pesquisas relacionadas à tomada de consciência das ações (Piaget, 1977) apontam para a importância de intervenções através de atividades que promovam a modificação de comportamentos. Neste caso, até mesmo a simples leitura, por parte dos alunos, dos questionários com as características citadas acima é mais uma forma de torná-los cientes dos pressupostos didático-pedagógicos que fundamentam nossas ações.

Observamos que a maior parte dos alunos nunca havia trabalhado com o software, o que demandou um tempo específico para o esclarecimento de dúvidas de ordem tecnológica, especialmente na construção de gráficos, necessários à análise e elaboração de comentários e justificativas solicitadas. Para redirecionar o tempo de envolvimento dos alunos às questões relacionadas ao conhecimento matemático, a partir da segunda edição do programa, procuramos aperfeiçoar o material de apoio, acrescentando orientações de acordo com questionamentos apresentados na anterior.

Como resultados em relação ao desempenho dos estudantes participantes, o percentual de aprovação foi de 53\%, 83\% e 64\%, respectivamente, na primeira, segunda e terceira edição do programa. Constatamos, também, que o período que propiciou um maior envolvimento dos estudantes foi o período de férias, confrontando as experiências realizadas em diferentes períodos acadêmicos. Destacamos que em período de recesso escolar os estudantes estiveram diariamente presentes no ambiente, o que promoveu uma qualidade diferenciada nas produções e nos conceitos de aprovação.

É importante destacar que mesmo buscando a coerência e a unidade entre os elementos que compõem o ambiente de aprendizagem do Mecam, as dissonâncias e diversidades precisam ser consideradas. Queremos dizer com isso que, embora busquemos propiciar trocas heterárquicas, desenvolvimento de autonomia, múltiplas formas de interação e problematização, encontramos momentos de dificuldades e de desencontros fazendo parte do processo. Tais dificuldades e desencontros estão relacionados ao uso da tecnologia, às interações entre os sujeitos, ao entendimento da aprendizagem como reprodução, ao medo de errar, à resistência e dificuldade em expressar suas idéias a partir da escrita, dentre outros.

De qualquer forma consideramos que, talvez mais importante do que se constituir numa opção de ensino à distância, seja a proposta metodológica deste programa, que permite gerar um processo reflexivo que leve os estudantes a assumirem sua parcela de responsabilidade por sua aprendizagem, desenvolvendo autonomia e, conseqüentemente, a capacidade de aprender a aprender.

\section{Considerações Finais}

É importante destacar que, nesse estudo, o suporte tecnológico foi o que permitiu a realização dos diálogos promovidos e conseqüentes resultados. Isto porque os alunos e professora estavam distantes fisicamente, o que não impediu a constituição dos diálogos sobre o aprender e os diálogos matemáticos. Além disto, existem especificidades importantes a comentar; uma delas é que os diálogos construídos de uma forma escrita possibilitam ao professor melhores condições de avaliação da aprendizagem dos alunos já que ele pode retomar os processos de pensamento em vários momentos; outro fator é um gradiente de socialização das reflexões feitas já que os percursos de raciocínio ficam disponíveis aos colegas podendo ser tomados em suas próprias reflexões, o que amplia a rede do diálogo matemático; a extensão do diálogo em tempos para além dos encontros presenciais.

Talvez tudo o que foi desenvolvido, no modo proposto neste estudo, possa ser 
feito sem o apoio de um ambiente virtual de aprendizagem. No entanto, há indicativos de que, com esse apoio, é possível a inclusão de recursos que possibilitem uma coconstrução, co-operação, co-autoria. Além disso, o registro dinâmico no fórum, podendo ser modificado, acrescentado, transformado ao longo do processo em produções coletivas, revela o texto dialógico que, de acordo com Freire (2001), constitui-se em fonte de aperfeiçoamento e desenvolvimento contínuos.

O levantamento dos resultados e a análise das interações sugeriram que os alunos têm grande dificuldade de ler e de interpretar, o que gera problemas de expressão clara e objetiva sobre os temas em estudo. É necessário incentivar a cooperação, a partir de estratégias que os levem a leituras e discussões de textos matemáticos, bem como a reflexões sobre o seu próprio fazer. Com efeito, o mais importante é a concepção de aprendizagem, de conhecimento, de novas relações professor-aluno.

A releitura e discussão dos resultados, com base nos pressupostos teóricos assumidos, nos permitem destacar como fatores positivos no desenvolvimento do programa:

$\checkmark$ a boa receptividade do mesmo, por parte dos estudantes;

$\checkmark$ o software Scientific Notebook, utilizado no programa, que possibilitou todas as formas de expressão da linguagem matemática: a verbal, por ser um editor de textos, a numérica e a geométrica, por ser processador numérico e gráfico, além da analítica com representação da simbologia matemática na mesma forma apresentada pelos livros de Cálculo e que os estudantes utilizam ao escrever em suas anotações;

$\checkmark$ o reconhecimento, por parte dos professores de matemática do Departamento de Matemática e Estatística, do valor acadêmico do programa;

$\checkmark$ o prêmio de melhor trabalho do CCET apresentado no Jovem Pesquisador de 2003, um estímulo e uma indicativo de confirmação de nossas hipóteses;

$\checkmark$ o caráter abrangente e integrador do programa, pelas professoras responsáveis e pelos bolsistas, com bolsa de iniciação científica ou voluntários, que propiciou um clima de interesse de estudantes e professores em conhecer o ambiente e a prática metodológica do Mecam;

$\checkmark$ o interesse manifestado pelos coordenadores dos cursos de Matemática, Computação e das Engenharias Mecânica e Química, na busca dentre alternativas burocráticas viáveis, por aquela que melhor se adequasse ao sistema da Universidade e permitisse, assim, a realização dos cursos, em caráter experimental;

$\checkmark$ o interesse demonstrado por estudiosos de Matemática ou de outras áreas, em todos os eventos onde divulgamos o programa, que possibilitou a discussão de temas de interesse do mesmo, junto a comunidades científicas.

Finalmente, destacamos que este trabalho pode contribuir para pensar a formação de professores. Uma possibilidade é utilizar o Mecam em parceria com a licenciatura em Matemática. Os professores precisam estar cientes de que não basta o conhecimento dos conteúdos para promover a aprendizagem de seus conceitos fundamentais. É preciso estar atentos a como pode ser construído o conhecimento, quais as condições para que a aprendizagem ocorra.

Destacamos também que, talvez mais importante do que se constituir numa opção de ensino à distância, seja a proposta metodológica deste programa, que permite gerar um processo reflexivo que leve os estudantes a assumirem sua parcela de responsabilidade por sua aprendizagem, desenvolvendo autonomia e, conseqüentemente, a capacidade de aprender a aprender.

\section{Referências bibliográficas}


CASTORINA, J. A. et al. Alcances do método de exploração crítica em psicologia genética. In: Psicologia genética: aspectos metodológicos e implicações pedagógicas. Tradução de José Cláudio de Almeida. Porto Alegre: Artes Médicas, 1988.

DEMO, P. Saber pensar. 2. ed. São Paulo: Cortez, Instituto Paulo Freire, 2001.

FREIRE, P., FREIRE, A.M.A. (Org.). Pedagogia dos sonhos possíveis. São Paulo: Editora Unesp, 2001.

LIMA, I.G. A equilibração dos processos cognitivos na aprendizagem de matemática no ambiente do Mecam. 2004, 220f. Tese (Doutorado em Informática em Educação) - PGIE, UFRGS, Porto Alegre, 2004.

LIMA, I. G., SAUER, L. Z., SOARES, E. M. S. Discutindo alternativas para ambientes de aprendizagem de matemática para cursos de Engenharia. In: World Congress on Engineering and Technology Education, 2004, Guarujá. Anais... Engineering Education in the Changing Society, Guarujá Council of Researches in Education and Sciences, 2004, p. 1159-1162.

LIMA, I. G., SAUER, L. Z., SOARES, E. M. S. Ambientes virtuais: espaços on-line de apoio á aprendizagem de matemática para engenharia. In: XXXIII Congresso Brasileiro de Ensino de Engenharia. Anais... Campina Grande, 2005.

LIMA, I. G., SAUER, L. Z., SOARES, E. M. S. Avaliação formativa: estratégia pedagógica para aprendizagem significativa de matemática em ambientes virtuais. In: 12o Congresso Internacional de Educação a distância. Anais... Florianópolis, 2005.

MATURANA, H. Emoções e linguagem na educação e na política. Belo Horizonte: Ed. da Ufmg, 1999.

PIAGET, J. A equilibração das estruturas cognitivas: problema central do desenvolvimento. Rio de Janeiro: Zahar, 1976.

PIAGET, J. A tomada de consciência. São Paulo: Melhoramentos, Edusp, 1977.

PONTE, J. P.; OLIVEIRA, H.; VARANDAS, J. M. (2003). O contributo das tecnologias de informação e comunicação para o desenvolvimento do conhecimento e da identidade profissional. In: FIORENTINI, D. (Ed.). Formação de professores de matemática: explorando novos caminhos com outros olhares. (159-192) Campinas: Mercado de Letras. Disponível em: http://www.educ.fc.ul.pt/docentes/jponte/. Acesso em agosto de 2005.

SAUER, L.Z. O diálogo matemático e o processo de tomada de consciência da aprendizagem em ambientes telemáticos. 2004, 195f. Tese (Doutorado em Informática em Educação) - PGIE, UFRGS, Porto Alegre, 2004.

SAUER, L. Z. e SOARES, E. M. Um novo olhar sobre a aprendizagem de Matemática para a Engenharia. In: CURY, H.N.(Org.). Disciplinas matemáticas em cursos superiores: reflexões, relatos, propostas. Porto Alegre: EDIPUCRS, 2004, p. 245270 . 
SOARES, E. M. S. Comportamentos matemáticos e o ensino de matemática para cursos de engenharia. 1997. 251 f. Tese (Doutorado em Metodologia do Ensino Superior) - Faculdade de Educação, Universidade Federal de São Carlos, São Carlos, 1997.

SKOVSMOSE, O. Cenários para Investigação. Boletim de Educação Matemática, Rio Claro, ano 13, n. 14, p. 66-91, 2000.

VALENTINI, C. B. Tecendo e aprendendo: redes sociocognitivas e autopoiéticas em ambientes virtuais de aprendizagem. 2003, 213f. Tese (Doutorado em Informática em Educação) - PGIE, UFRGS, Porto Alegre, 2003. 